\title{
Wireless Integrated Voltametric and Amperometric Biosensing
}

\author{
${ }^{1}$ Mohsen Mollazadeh, ${ }^{1}$ Kartikeya Murari, ${ }^{1}$ Christian Sauer, ${ }^{2}$ Milutin Stanac̀evic̀, ${ }^{1}$ Nitish Thakor, \\ ${ }^{3}$ Gert Cauwenberghs \\ ${ }^{1}$ Department of Biomedical Engineering, Johns Hopkins University, Baltimore. \\ ${ }^{2}$ Department of Electrical Engineering, State University of New York, Stony Brook. \\ ${ }^{3}$ Department of Neurobiology, University of California, San Diego. \\ mohsenm@jhu.edu, kartik@jhu.edu, csauer@jhu.edu, milutin@ece.sunysb.edu, \\ nitish@jhu.edu, gert@ucsd.edu
}

\begin{abstract}
Central Nervous System (CNS) uses the interplay between signals of different modalities to transfer and process information. Neurological events are characterized by changes in both neurochemical concentrations and the electrical activity of neurons. Electrophysiological and neurochemical events are highly correlated as one causes the other and vice-versa. The ability to simultaneously record electrical and chemical activity is of considerable research and diagnostic importance. Here, we present a hardware implementation for wireless power and clock transfer to and serial transmission of digitized neurochemical and electrophysiological data from sensors over one RF link. The idea can be extended to sensors of different modalities having widely different data rates. Neurochemical data are acquired in real-time from a custom multichannel very large scale integrated (VLSI) potentiostat chip at $5 \mathrm{~Hz}$. Field potential data were sampled at $400 \mathrm{~Hz}$. A custom VLSI chip powers up and supplies clocks to the potentiostat and telemeters the multiplexed data. All the chips were fabricated in AMI 3M-2P 0.5 $\mu$ CMOS process. We demonstrate successful operation of the system with wireless powering and telemetry of the multiplexed data.
\end{abstract}

\section{INTRODUCTION}

Simultaneous detection and sensing of neurochemicals and electrophysiological field potentials would be very useful in studying the interaction between the chemical synaptic activity and the electrical neuronal activity. At the insulating gap between two neurons (the synapse), the electrical activity in the pre-synaptic neuron causes the release of neurotransmitters into the synaptic cleft. Post-synaptic neurons sense these neurotransmitters and based on the specific chemical message initiate or suppress the transmission of electrical activity through them. To be able to monitor these related signals in-vivo is even more useful as it allows continuous sensing from awake and behaving animals. This could provide important information regarding neurological conditions where there is an imbalance between the chemical and electrical activity such as epilepsy [1].

\section{SYSTEM DESIGN AND DATA TIMING}

\section{A. VLSI Chips}

Our system consists of two custom designed and fabricated VLSI chips. A multichannel VLSI potentiostat [2] is used

This work was supported by NIH MH062444 065296, DARPA, Army Research Office and the Whitaker Foundation. Chips were fabricated through the MOSIS foundry service.

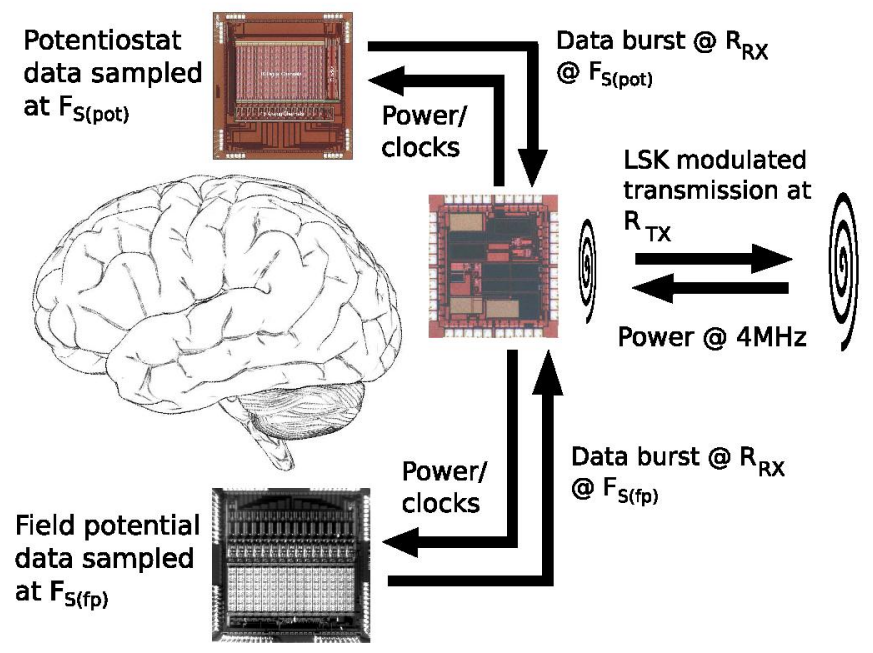

Fig. 1. System diagram showing micrographs of the individual chips in an eventual implantable scenario. The field potential chip is under testing. In this work, field potential data are played from a memory.

as a back-end to an electrochemical neurotransmitter sensor that transduces concentrations into currents. The $9 \mathrm{~mm}^{2}$ chip acquires 16 channels of currents potentiostatically and implements a configurable $\Delta-\Sigma$ analog-to-digital converter (ADC) to convert them into a serial bit-stream and consumes $200 \mu \mathrm{W}$ per channel. The power harvesting and telemetry chip [3] uses a RF link to transfer power at a biologically optimum frequency of $4 \mathrm{MHz}$ [4]. It has circuitry to generate a regulated power supply and recover clocks for the sensor chips. Data generated by the sensor chips is LSK modulated and transmitted over the same link. The $0.1 \mathrm{~mm}^{2}$ chip dissipates about $35 \mu \mathrm{W}$ of power and can supply $6 \mathrm{~mW}$ of power and clocks wirelessly across $25 \mathrm{~mm}$.

\section{B. Timing}

Neurochemical changes in general are on a very different time scale (on the order of several hundreds of milliseconds to seconds) than field potential events (on the order of a few tens of milliseconds). This neccesitates widely different sampling frequencies for the two signals. For the VLSI potentiostat, sampling frequency depends on the concentration of the neurotransmitters being measured (lower concentrations need a longer conversion time which implies a lower 


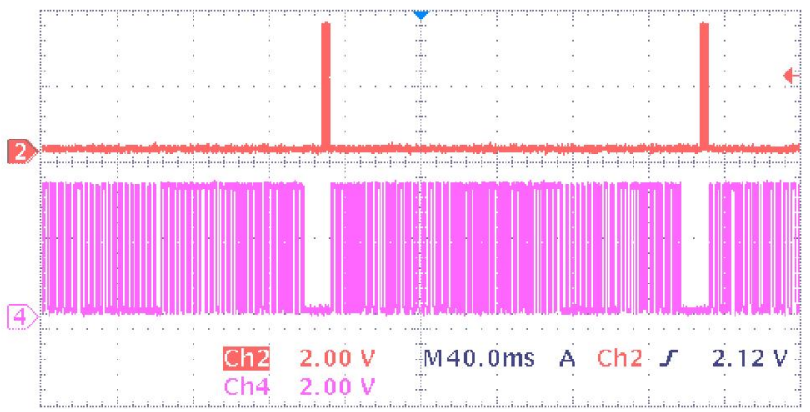

Fig. 2. Scope plot showing the timing of our scheme. The top trace shows potentiostat data bursts. Lower trace shows the transmission of the multiplexed potentiostat and field potential data.

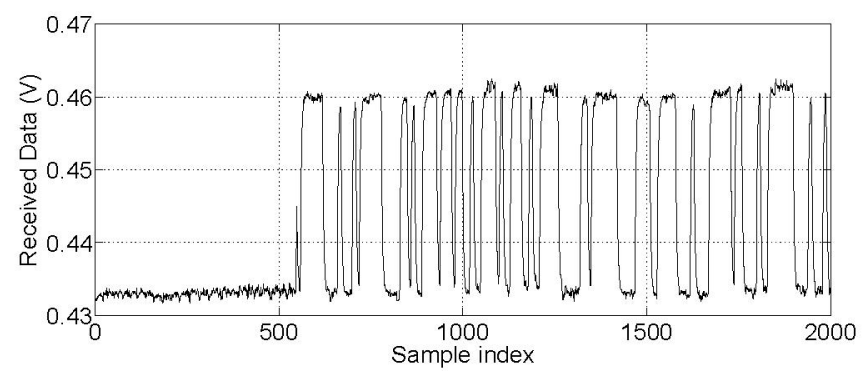

Fig. 3. Portion of the data received the base station. It is a zoomed view of the data in the lower trace of Fig. 2 after its transmission, reception, decoding and acquisition through a DAQ card.

sampling frequency). The chip digitizes 16 channels of the neurotransmitter concentration at 16 bits per channel 5 times every second and serially outputs the data at a burst rate of $R_{R X}=64 \mathrm{KHz}$. The field potential data are sampled at $400 \mathrm{~Hz}$ and digitized to 12 bits per sample. The wireless telemetry chip has a bandwidth limitation because it utilizes the same link for power and data transmission. The Q-factor of the coils constrains the characteristics of the system. The Qfactor is proportional to the amount of power that can be transfered, and inversely proportional to the bandwidth. We choose a rate of $4 \mathrm{kbps}$. The timing diagram of our scheme is shown in Fig. 2. At a wireless transmission rate of 4 kbps, potentiostat data transmission takes $16 \times 16 / 4 \mathrm{k}=64$ ms. During this time, the field potential data are stored in a memory and transmitted after the potentiostat data.

\section{RESULTS AND CONCLUSIONS}

A modified class-E amplifier is used to drive the transmission coil and provide power for the system. A class E amplifier is used because of its high efficiency and low power supply voltage requirement [3]. System clock and supply voltages are recovered through the wireless chip.

Fig. 3 shows the recovered data at the transmitter side. The current through the coil is converted to a voltage through a current sensing transformer. The voltage is then put through a rectifier and filtered to recover the envelope. A data acquisition card samples the voltage at the output of the envelope detector for further processing. The amplifier and

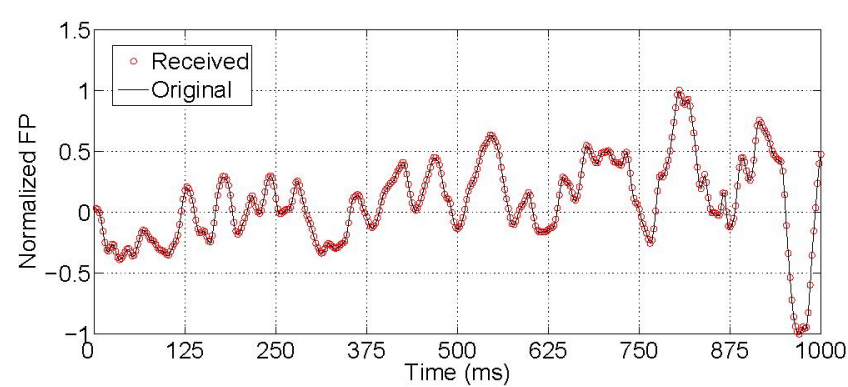

(a)

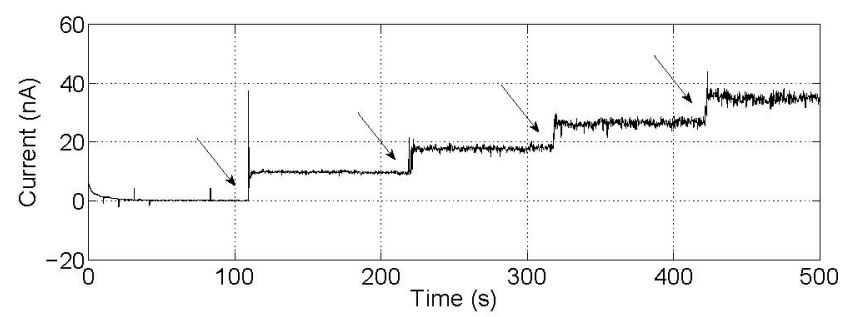

(b)

Fig. 4. (a) Original and demultiplexed field potential (FP) data shown for a 1 second window. (b) Demultiplexed output of one potentiostat channels showing response to the addition of $500 \mu \mathrm{M}$ boluses of the neurotransmitter dopamine to the test solution.

data recovery circuit were realized with discrete components on a board separate from the microchip test circuitry.

Fig. 4 shows the decoded data for both modalities of the signal. The potentiostat was used to sense the neurotransmitter dopamine with a commercial electrode. The experimental protocol is described elsewhere [2]. Arrows denote times of dopamine addition to test solution. In order to show the feasibility of transmission, the field potential data were previously sampled at $400 \mathrm{~Hz}$ from a human subject using a 10-20 electrode system, stored in a memory and multiplexed with the potentiostat data prior to transmission.

We were able to achieve simultaneous telemetry of electrophysiological and neurochemical data while supplying the sensor chips with power and clocks wirelessly. The system consumed less than $4 \mathrm{~mW}$ of power running off $3.3 \mathrm{~V}$. There were no bit errors at our chosen transmission frequency.

We have presented a novel integrated system for simultaneous transmission of electrophysiological and neurochemical signals. In the near future, we plan to integrate all the systems on a standalone silicon die that can be used in a fully-implantable system.

\section{REFERENCES}

[1] Fried, I. et. al., "Cerebral microdialysis combined with single-neuron and electroencephalographic recording in neurosurgical patients," $J$. Neurosurg., vol. 91:4, 1999, pp 697-705.

[2] Murari, K. et. al., "Integrated potentiostat for neurotransmitter sensing," Engineering in Medicine and Biology Magazine, IEEE, vol. 24:6, 2005, pp 23-29.

[3] Sauer, C. et. al., "Power harvesting and telemetry in CMOS for implanted devices," IEEE Trans. Circuits and Systems (I), vol. 52:12, 2005 , pp $2605-2613$.

[4] Vaillancourt, P. et. al., " EM radiation behavior upon biological tissues in a radio-frequency power transfer link for a cortical visual implant," IEEE Eng. in Med. and Biol. Conf., vol. 6, pp 2499-2503, 1997. 